\title{
Dermatophytosis in a Dog Seropositive for Ehrlichia Spp
}

\author{
Peda $\mathrm{A}^{1}$, Betance $\mathrm{L}^{1}$, Soto $\mathrm{E}^{3}$, Fraites $\mathrm{T}^{2}$ and Verma $\mathrm{A}^{* 3}$ \\ ${ }^{1}$ Department of Clinical Sciences, Ross University School of Veterinary Medicine, Basseterre, St. Kitts \\ ${ }^{2}$ Laboratory services, Ross University School of Veterinary Medicine, Basseterre, St. Kitts \\ ${ }^{3}$ Department of Biomedical Sciences, Ross University School of Veterinary Medicine, Basseterre, St. Kitts
}

${ }^{*}$ Corresponding author: Verma A, Department of Biomedical Sciences, Ross University School of Veterinary Medicine, P.O. Box 334, Basseterre, St. Kitts, Fax: +1 869466 5206, Tel: +1 8694654161 x 1230, E-mail: averma@rossvet.edu.kn

Citation: Peda A, Betance L, Soto E, Fraites T, Verma A (2014) Dermatophytosis in a Dog Seropositive for Ehrlichia Spp. J Vet Sci Anim Husb 2(2): 201. doi: 10.15744/2348-9790.1.501

Received Date: June 21, 2014 Accepted Date: August 11, 2014 Published Date: August 13, 2014

\begin{abstract}
In this report, we present a case of dermatophytosis in a dog seropositive for Ehrlichia spp.Mild annular skin lesions observed on initial presentation persisted and became worse during the course of antibiotic therapy. Microscopic examination of the fungal culture of skin scrapings revealed infection with Microsporum gypseum. The patient fully recovered following treatment with topical $1 \%$ clotrimazole and antifungal shampoo.
\end{abstract}

Keywords: Dermatophytosis; Microsporum gypseum; Dog; Ehrlichiosis

\section{Introduction}

Dermatophytosis is a common zoonotic fungal infection of the keratinized structures such as superficial skin, hair, feathers, horn, claws and nails. Microsporum and Trichophyton are the two genera of dermatophytes that affect animals. Dermatophytes grow best in warm and humid environments and are, therefore, more common in tropical and subtropical regions [1].

The dermatophytes are classified on the basis of their reservoirs as zoophilic, anthropophilic and geophilic. Zoophilic and anthrophilic are primarily found in animals and humans, respectively. Geophilic are found in soil, where they live on decomposing keratinous material, and can infect both humans and animals. Transmission occurs by direct contact with a host, direct or airborne contact with its hairs or skin scales, or directly from the soil. Infective spores on hair or dead skin cells can remain viable for several months to years in the environment $[2,3]$.

Predisposing factors for many fungal infections include, among others, poor nutritional status and disregulated immune response $[4,7]$. Dogs with ehrlichiosis, a bacterial infection of monocytic or granulocytic cells, can easily become infected with a variety of secondary pathogens [8]. In this report, we discuss a case of cutaneous mycosis in a dog, caused by Microsporum gypseum, following an infection with Ehrlichia spp.

\section{Case report}

A two-year-old intact female pit bull mix dog was presented to the Ross University School of Veterinary Medicine's veterinary clinic by the Saving Animals from Emergency on St. Kitts (SAFE) program. The dog was found in lateral recumbency on a road in a severely emaciated condition. The animal was dehydrated, slightly pyrexic $\left(102.7^{\circ} \mathrm{C}\right)$ and had numerous ticks and fleas on the body with pressure sores of varying sizes on her right and left hip. There was alopecia over her right lateral hock and small circular alopecic areas on her pinnae and limbs. The animal also had generalized muscular atrophy.

Initial diagnostic tests revealed severe anemia with a packed cell volume (PCV) of 15\% with no regenerative indicators, thrombocytopenia (platelet count of 62,000; no macro platelets but elevated mean platelet volume), and high blood urea nitrogen (BUN of 38). Snap 4Dx plus test (Idexx laboratories, Westbrook, ME, USA) was positive for Ehrlichia spp. While Snap Parvo test (Idexx laboratories) was negative.The animal was hospitalized and given supportive care with crystalloid replacement intravenous fluid therapy, gastro protectants(famotidine $0.2 \mathrm{mg} / \mathrm{kg}$, bid, iv), nitenpyram (11.4 mg, po) for fleas, pyrantal pamoate (150mg, repeated in 2 weeks) for suspect intestinal parasites, and doxycycline (150 mg daily for 21 days) for ehrlichiosis. Ticks were manually removed. The dog was discharged next day after improvement in clinical signs. 
The patient returned 2 days later for decreased appetite and vomiting. She was lethargic and had pale mucous membranes. Blood glucose was $22 \mathrm{mg} / \mathrm{dl}, \mathrm{PCV}$ was $23 \%$, and systolic blood pressure was $50 \mathrm{mmHg}$. She was hospitalized and supportive care for hypotension and hypoglycemia was instituted. She responded to treatments and was discharged 5 days later.

Twenty-eight days after the initial presentation, the patient returned with a complaint of worsening skin lesions with itching and hair loss. Physical exam revealed multiple skin lesions that were annular, alopecic, erythematous and scaly with peripheral crusting. Skin lesions were present on left lateral neck ( $4 \mathrm{~cm}$ in diameter; Figure 1A), right lateral shoulder $(2.5 \mathrm{x} 1.5 \mathrm{~cm})$, right lateral thigh ( $3 \mathrm{~cm}$ in diameter), and distal one-third of tail (Figure 1B).Ringworm was suspected because of typical clinical presentation and a history of poor-nutrition and immunosuppression. Skin scrapings and hair from the periphery of the lesions were gently implanted into the surface of dermatophyte test medium (DTM; Shelby scientific,Macomb, MI) and incubated at $25^{\circ} \mathrm{C}$ (room temperature). An alkaline reaction on the DTM and a moldy fungal growth were observed. A part of fungal colony was stained with lactophenol cotton blue and examined microscopically. Several thin-walled, macroconidia were observed. Macroconida were broad in the middle but round at the ends, showing a 'boat-shape' morphology that is characteristic of Microsporum gypseum. No microconidia were observed (Figure 2). A shampoo containing 1\% ketoconazole and $2 \%$ chlorhexadine (day 1, 3, 8, then 7days) was added to the treatment regimen. Topical clotrimazole $1 \%$ (bid to lesions) was also added 1 week later after confirmation of $M$. gypseum by culture. The patient showed continued improvement of skin lesions and complete resolution after 30 days.
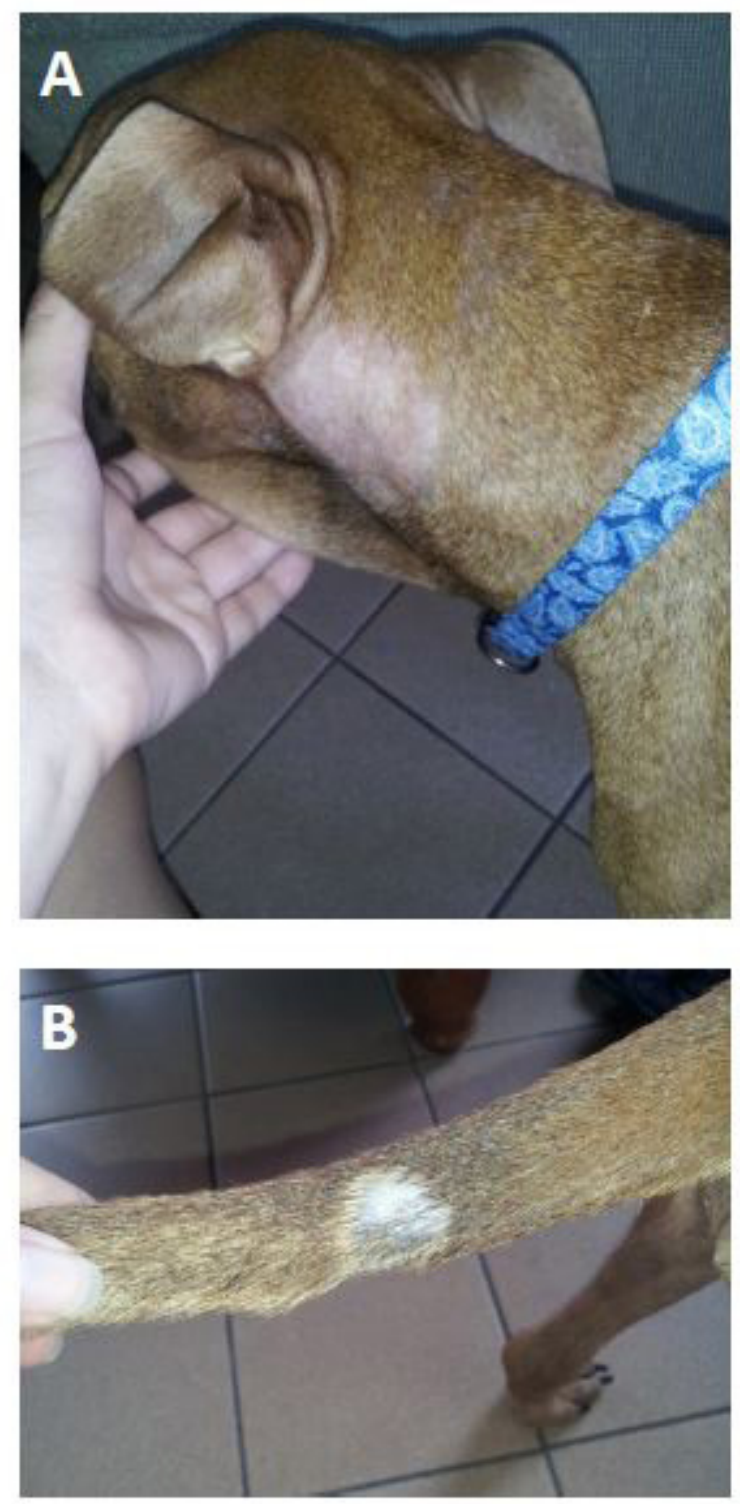

Figure 1: Lesions in a dog with dermatophytosis. Typical annular, alopecic, and scaly skin lesions on left lateral neck (A), and distal one-third of tail (B). 


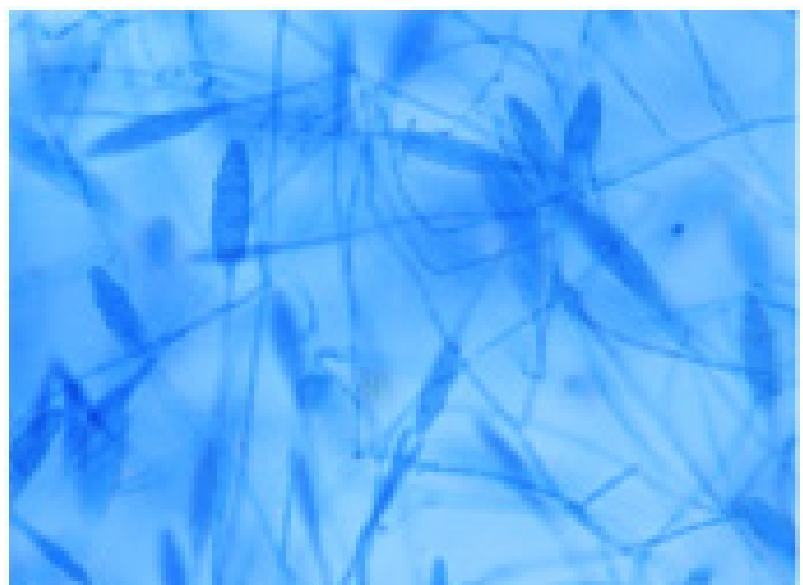

Figure 2: Microsporum gypseum. Lactophenol cotton blue mount from dermatophyte test medium. Macroconidia are thin-walled and have characteristic 'boat-shape'. Microconidia are absent. 400X

\section{Discussion}

Although a species-specific serological test was not performed, the clinicopathological picture and the absence of Amblyomma americanum, the only proven vector for transmission of E ewingii, on this island is suggestive of E. canis infection [5,6]. Microsporum gypseum is a geophilic dermatophyte that is commonly associated with skin infections in tropical and subtropical areas. Dogs are exposed to the fungal spores through digging and rooting in the contaminated areas. Since dermatophytosis is a pleomorphic disease, it cannot be diagnosed solely on the clinical signs [1,3]. For a definitive diagnosis, identification by a fungal culture or a molecular technique is required. In this patient, we cultured and microscopically identified the characteristic macroconidial morphology of M. gypseum.

Widespread skin lesions, as seen in this case, indicate an underlying systemic condition. In a previous study, generalized, chronic dermatophytosis was observed in dogs concurrently suffering from leishmaniosis +/erhlichiosis or diabetes mellitus [7]. In this patient, a number of debilitating factors, including an exposure to Ehrlichia spp. might have contributed towards predisposition of the animal to generalized dermatophytic infection.

\section{Acknowledgements}

We thank Gillian Carmichael-Branford, Julienne Rawlins and Iona Simmonds for technical assistance.

\section{References}

1. Green CE (2006) Cutaneous fungal infections: Infectious diseases of the dog and cat, $3^{\text {rd }}$ edition, Saunders Elsevier, St. Louis, Missouri, USA.

2. Ellis D, Marriott D, Hajjeh RA, Warnock D, Meyer W, et al. (2000) Epidemiology: surveillance of fungal infections. Med Mycol 38: $173-82$.

3. Moretti A, Agnetti F, Mancianti F, Nardoni S, Righi C, et. al. (2013) Dermatophytosis in animals:epidemiological, clinical and zoonotic aspects. G Ital Dermatol Venereol 148: 563-72.

4. Scher RK, Rich P, Pariser D, Elewski B (2013) The epidemiology, etiology, and pathophysiology of onychomycosis. Semin Cutan Med Surg. 32: S2-4.

5. Kelly P, Lucas H, Beati L, Yowell C, Mahan S, et al. (2010) Rickettsia africae in Amblyomma variegatum and domestic ruminants on eight Caribbean islands. J Parasitol 96: 1086-8.

6. Little SE (2010) Ehrlichiosis and anaplasmosis in dogs and cats. Vet Clin North Am Small Anim Pract 40: 1121-40.

7. Cerundolo R (2004) Generalized Microsporum canis dermatophytosis in six Yorkshire terrier dogs. Vet Dermatol 15: 181-7.

8. Green CE (2006) Canine monocytotropic ehrlichiosis and neorickettsiosis: Infectious diseases of the dog and cat, $3^{\text {rd }}$ edition, Saunders Elsevier, St. Louis, Missouri, USA.

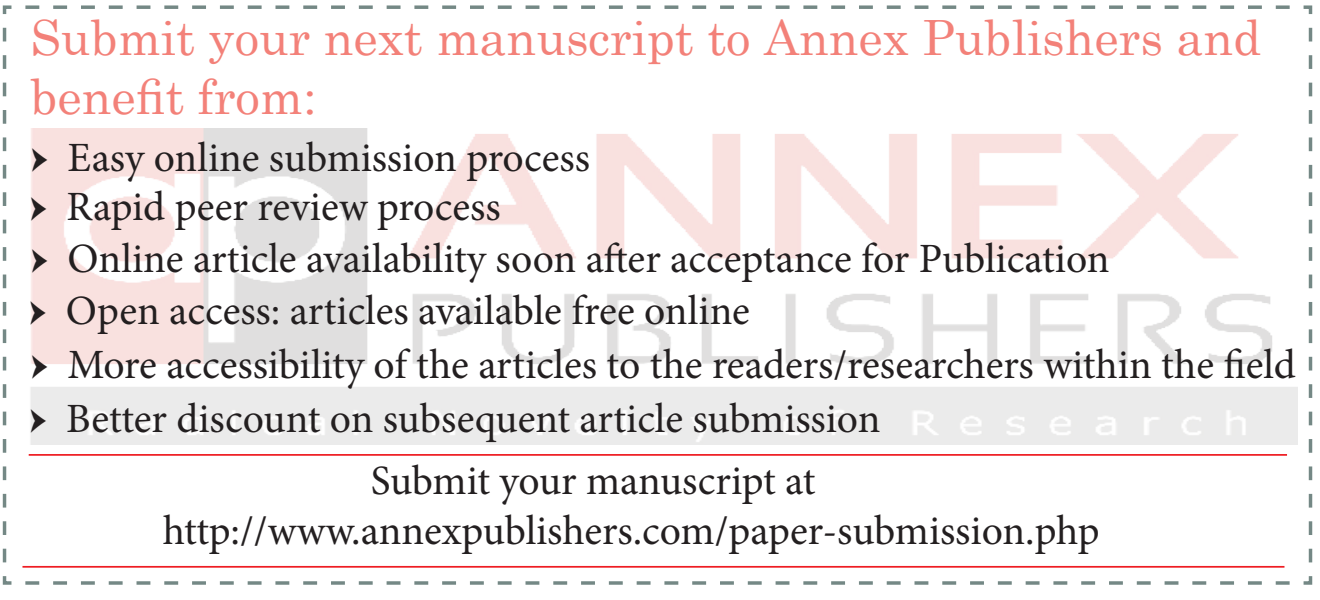

